\title{
Pigmented fungiform papillae of the tongue: clinical and dermoscopic features
}

\author{
Niema Aqil, Mounia Bennani, Aicha Nassiri, Mariame Meziane, Salim Gallouj, \\ Fatima Zahra Mernissi
}

Department of dermatology, Hassan II University Hospital Center, Fes, Morocco

Corresponding author: Dr. Niema Aqil, E-mail: niemaaqi190@gmail.com

Sir,

We present a clinical report of pigmented fungiform papillae of the tongue (PFPT), describing the dermoscopic pattern of the lesions. This case is of significance because, although PFPT is not uncommon, it is not present as an entity in most textbooks and seldom mentioned in the medical literature. Our aim is to emphasise that a prompt diagnosis will avoid further unnecessary investigations.

A 12-year-old Moroccan girl consulted our department of dermatology for multiple erythematous and hyperpigmented papules, small and asymptomatic with the tip of the tongue evolving for 5 years. The patient was not taking any medication and was in good general health. The parents did not present similar pigmentation of the oral mucosa. She was of phototype IV. Examination of the oral mucosa showed pigmentation limited to the fungiform papillae of the dorsum of the tongue (Fig. 1).

The majority of the fungiform papillae were pigmented and were present in a diffuse, symmetrical pattern, predominantly on the tip and lateral aspects of the dorsum of the tongue (Fig. 2). The fungiform papillae in the central area were not pigmented.

Dermatoscopy showed that the small papules corresponded to enlarged fungiform papillae and showed linear and pointed vessels in their central part and a pinkish collar around. Some papules were pigmented in their central part and in particular at the edge of their central part with the presence of an unpigmented collar around the central pigmentation and and dichotomized vessels that originate at the base, resembling a rose petal appearance.

Complete physical examination was normal including eyes, nails, and genitals. Laboratory values (basic metabolic panel, complete blood count, iron test, and anti nuclear antibodies) showed no alterations.

The diagnosis of racial pigmented fungiform papillae of the tongue was considered. The patient was reassured of the benign nature of the condition and no treatment was given.

The pigmented fungiform papillae of the tongue correspond to a benign change in the tongue characterized by a hyperpigmentation limited to fungiform papillae giving a dotted appearance. This pigmentation usually develops in black skinned subjects in childhood or in young adults. The lesions are classically asymptomatic. Dermatoscopy shows an enlargement of the fungiform papillae with dilated vessels and pigmentation that give a "rose petal" appearance [1]. An additional element for the identification of this entity is the presence of an unpigmented collar around the central pigmentation.

The pathogenesis is unknown, but it has been hypothesized that pigmentation may develop as a result of inflammation. In fact, the cases that were biopsied showed melanophages pigmented in the lamina propria [2-4] as a possible consequence of a passage of melanin from the epithelium to the lamina propria following an inflammation that would reach the basal layer of the lamina propria [2-4]. This hypothesis is supported by the presence in dermatoscopy of dilated

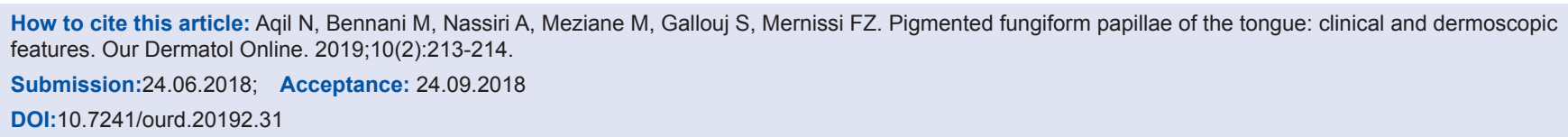




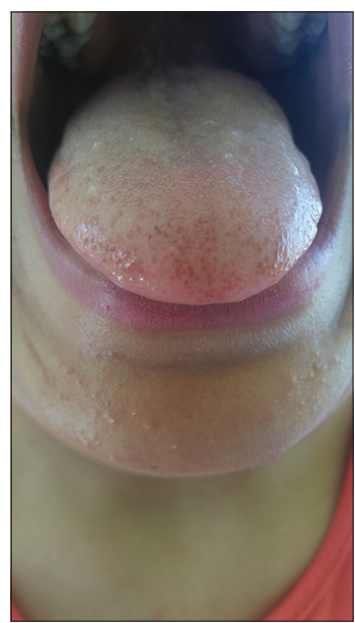

Figure 1: Pigmentation limited to the fungiform papillae of the dorsum of the tongue.

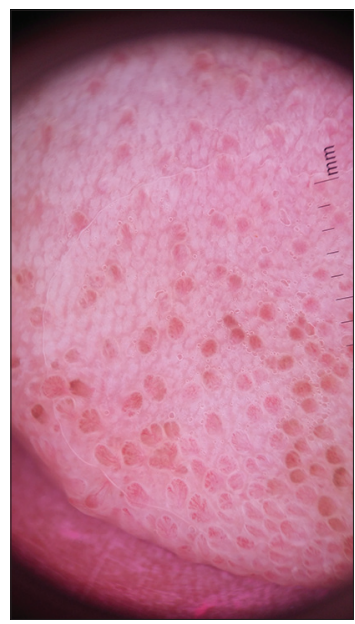

Figure 2: Dermoscopy of the peigmented fungiform papillae.

vessels inside the papillae (sign of inflammation) and by a pigmentation limited to the central part of the papillae (corresponding to the surface projection of the lamina propria) and not in the peripheral collar (corresponding to the projection of the only epithelium) [5]. Differential diagnoses of pigmented fungiform papillae of the tongue include amalgam tattoo, Peutz-Jeghers syndrome, melanocyte tumors and Addison's disease where pigmentation is not limited to fungiform papillae.

As it is a benign condition there is no need to treat it, neither has any treatment been reported. No malignant transformation of PFPT has been described. Today, increased public awareness of the malignant potential of pigmented skin lesions has led to an increasing number of consultations. Clinicians unfamiliar with common oral and tongue changes may suggest that these alterations are pathological, leading to unnecessary investigations and dispensable biopsies. Non-invasive imaging devices like dermoscopy can avoid these unnecessary procedures.

\section{CONSENT}

The examination of the patient was conducted according to the Declaration of Helsinki principles.

\section{REFERENCES}

1. Mukamal LV, Ormiga P, Ramos-E-Silva M. Dermoscopy of the pigmented fungiform papillae of the tongue. J Dermatol. 2012;39:397-9.

2. Holzwanger JM, Rudolph RI, Heaton CL. Pigmented fungiform papillae of the tongue: a common variant of oral pigmentation. Int J Dermatol. 1974;13:403-8.

3. Werchniak AE, Storm CA, Dinulos JGH. Hyperpigmented patches on the tongue of a young girl. Pigmented fungiform papillae of the tongue. Arch Dermatol. 2004;140:1275-80.

4. Lin Y-T, Chou C-L. Pigmented macule on the tongue of a 12-yearold girl. J Am Acad Dermatol. 2013;69:e229-30.

5. Cinotti E, Labeille B, Cambazard F, Perrot JL. [Dermoscopy and reflectance confocal microscopy examination of pigmented fungiform papillae of the tongue]. Ann Dermatol Venereol. 2016;144:323-5.

Copyright by Niema Aqil, et al. This is an open access article distributed under the terms of the Creative Commons Attribution License, which permits unrestricted use, distribution, and reproduction in any medium, provided the original author and source are credited.

Source of Support: Nil, Conflict of Interest: None declared. 